\title{
Noninvasive identification of left-sided heart failure in a population suspected of pulmonary arterial hypertension
}

\author{
Wouter Jacobs ${ }^{1,2}$, Thelma C. Konings ${ }^{3}$, Martijn W. Heymans ${ }^{4}$, Anco Boonstra', \\ Harm Jan Bogaard ${ }^{1}$, Albert C. van Rossum ${ }^{3}$ and Anton Vonk Noordegraaf ${ }^{1}$
}

Affiliations: ${ }^{1}$ Dept of Pulmonology, VU University Medical Center, Amsterdam, The Netherlands. ${ }^{2}$ Dept of Pulmonology, Martini Hospital, Groningen, The Netherlands. ${ }^{3}$ Dept of Cardiology, VU University Medical Center, Amsterdam, The Netherlands. "Dept of Epidemiology and Biostatistics, VU University Medical Center, Amsterdam, The Netherlands.

Correspondence: Anton Vonk Noordegraaf, VU University Medical Center, Dept of Pulmonary Medicine, PO Box 7057, 1007MB Amsterdam, The Netherlands. E-mail: a.vonk@ivumc.nl

ABSTRACT Exclusion of pulmonary hypertension secondary to left-sided heart disease (left heart failure $(\mathrm{LHF})$ ) is pivotal in the diagnosis of pulmonary arterial hypertension (PAH). In case of doubt, invasive measurements are recommended. The aim of the present study was to investigate whether it is possible to diagnose LHF using noninvasive parameters in a population suspected of PAH.

$300 \mathrm{PAH}$ and 80 LHF patients attended our pulmonary hypertension clinic before August 2010, and were used to build the predictive model. $79 \mathrm{PAH}$ and 55 LHF patients attended our clinic from August 2010, and were used for prospective validation.

A medical history of left heart disease, S deflection in V1 plus R deflection in V6 in millimetres on ECG, and left atrial dilation or left valvular heart disease that is worse than mild on echocardiography were independent predictors of LHF. The derived risk score system showed good predictive characteristics: $\mathrm{R}^{2}=0.66$ and area under the curve 0.93 . In patients with a risk score $\geqslant 72$, there is $100 \%$ certainty that the cause of pulmonary hypertension is LHF. Using this risk score system, the number of right heart catheterisations in LHF may be reduced by $20 \%$.

In a population referred under suspicion of $\mathrm{PAH}$, a predictive model incorporating medical history, ECG and echocardiography data can diagnose LHF noninvasively in a substantial percentage of cases.

@ERSpublications

A risk score system can identify left-heart failure as alternative $\mathrm{PH}$ cause in patients suspected of PAH clinically http://ow.ly/JAIkR

For editorial comments see Eur Respir J 2015; 46: 299-302 [DOI: 10.1183/13993003.00456-2015]

Received: Nov 022014 | Accepted after revision: Feb 192015 | First published online: April 022015

Conflict of interest: None declared.

Copyright OERS 2015 


\section{Introduction}

Pulmonary arterial hypertension (PAH) is a progressive disease of the pulmonary vasculature, leading to high morbidity, right heart failure and death within 2-3 years if left untreated $[1,2]$. While PAH is rare, pulmonary hypertension $(\mathrm{PH})$ secondary to left heart failure (LHF) is more common [3]. Right heart catheterisation (RHC) is recommended for final diagnosis when PAH is suspected clinically [4]. An invasively measured pulmonary capillary wedge pressure of $15 \mathrm{mmHg}$ and/or increased left ventricular end-diastolic pressure (LVEDP) are used to discriminate between LHF and PH from other causes. LHF is caused by chronic heart failure attributed to left ventricular systolic or diastolic dysfunction, or by valvular diseases [5]. $\mathrm{PH}$ with normal wedge pressure fits a diagnosis of $\mathrm{PAH}$, provided other causes of $\mathrm{PH}$ with normal wedge, including lung disease and chronic thromboembolic $\mathrm{PH}$, are excluded [6]. Increased $\mathrm{PH}$ awareness in combination with high LHF prevalence and, in particular, difficulties in diagnosing heart failure with preserved ejection fraction noninvasively have increased referrals to PAH centres and the number of RHCs performed [7]. A model to predict, in advance, the likelihood of LHF could decrease the burden to the patient and economic cost associated with these referrals. Although rare, complications of RHC, such as ventricular tachyarrhythmia, vascular or ventricular perforation, bleeding, pneumothorax, and even death, may occur [8].

The aim of this study was to develop a noninvasive predictive model of LHF as a cause of PH in a patient population referred to a $\mathrm{PH}$ centre, suspected of $\mathrm{PAH}$ clinically. Predictors of heart failure in patient medical history, ECG and echocardiography were used in order to identify referred patients in whom a firm diagnosis of LHF could be made noninvasively [9-13].

\section{Methods}

Study design and patients

The VU University Medical Centre is a referral centre for diagnosis and treatment of PH in Amsterdam, The Netherlands. All patients suspected of $\mathrm{PAH}$ and referred to our $\mathrm{PH}$ unit and in whom a diagnosis of $\mathrm{PAH}$ ( $\mathrm{PH}$ group 1) or $\mathrm{PH}$ secondary to LHF ( $\mathrm{PH}$ group 2) was made were included. The retrospective patient cohort attended our clinic between April 1998 and July 2010, and was used to build the predictive model. The patient cohort attending our clinic from August 2010 to December 2012 was used to prospectively validate our model.

All patients underwent RHC, since interpretation of the echocardiography was not conclusive for the diagnosis of $\mathrm{PH}$ secondary to left heart disease in this patient cohort. In all patients, $\mathrm{PH}$ was present based on a mean pulmonary artery pressure $\geqslant 25 \mathrm{mmHg}$. We used the following decision rule to discriminate between precapillary $\mathrm{PH}$ and $\mathrm{PH}$ secondary to LHF [14]. If wedge pressure was $>15 \mathrm{mmHg}$ at rest or increased $>15 \mathrm{mmHg}$ after $500 \mathrm{~mL}$ of saline infusion, the diagnosis of $\mathrm{PH}$ secondary to LHF was made [15]. If no reliable wedge was obtained, LVEDP was measured. In precapillary PH, chronic thromboembolic $\mathrm{PH}$ ( $\mathrm{PH}$ group 4) was ruled out by the combination of ventilation/perfusion scintigraphy and computed tomography (CT) angiography. $\mathrm{PH}$ due to parenchymal lung disease (PH group 3) was excluded by lung function testing and high-resolution CT; in addition, polysomnography was performed to exclude sleep disordered breathing.

Potential LHF predictors known from the literature were recorded from the medical history, ECG and echocardiographic parameters. Predictors recorded were: age; body mass index; sex; a medical history of hypertension, diabetes, dyslipidaemia or left heart disease (either coronary artery disease or left valvular heart disease that was worse than mild); and smoking history $>1$ pack-year. From ECG, the $\mathrm{S}$ deflection in V1 (SV1) in millimetres, the R deflection in V6 (RV6) in millimetres and ECG evidence of left atrial dilation (yes/no), and from the echocardiographic parameters, the echocardiographic presence of left atrial dilation (yes/no) or left valvular heart disease that was worse than mild (yes/no) were included in the analysis. Echocardiographic left atrial dilation was defined according to recommendations for chamber quantification from the American Society of Echocardiography [16]. Severity of valvular heart disease was assessed according to European Association of Echocardiography guidelines [17-19]. Echocardiographic parameters were scored by a cardiologist blinded to the diagnosis; ECG data were measured by an observer blinded to the definite diagnosis.

\section{Data analysis}

Patient characteristics in both groups were described. Continuous variables are presented as mean $\pm \mathrm{SD}$ and categorical data as $\mathrm{n}$ or $\%$.

\section{Model building}

The effect of the predictor variables was evaluated by univariate logistic regression. A potential nonlinear behaviour of continuous factors was tested and examined using restricted cubic spline functions and spline plots. These tests showed that the linearity assumption was not violated and that transformation of 
continuous variables was not necessary. We used multiple imputation to fill in variables with missing values by using the multivariate imputation by chained equation (MICE) procedure. This method estimates several plausible values to fill in the missing values in the variables. We imputed 10 data sets. Multivariable logistic regression with backward elimination determined the final model. Variable selection was done by taking into account the imputed data sets. This means that logistic regression models were estimated in each imputed data set, that regression coefficients and standard errors were pooled, and that the p-value of each coefficient in the multivariable model determined [20]. This step was repeated until variables with a p-value $<0.10$ were retained in the final model. Regression coefficients and standard errors were converted to odds ratios and corresponding $95 \%$ confidence intervals.

\section{Performance of the prediction model}

The performance of the prediction model was studied in terms of discrimination, explained variance and calibration. Discrimination expresses how well the prognostic model distinguishes between patients with LHF and PAH, and was obtained by the area under the receiver operating characteristic curve (AUC). The explained variance, calculated as Nagelkerke's $\mathrm{R}^{2}$, gives an indication of how much of the variance in the outcome can be explained by the predictors. To reflect how well predicted and observed probabilities agreed and to obtain an insight into the model's calibration, the calibration slope was calculated. The calibration slope can also be used as a shrinkage factor to shrink the regression coefficients. This is performed because prognostic models usually perform better in subjects used to build the model than in new subjects due to optimism in regression coefficients and performance measures [21]. To determine the shrinkage factor, an internal validation procedure using bootstrapping techniques was used. The AUC, explained variation and slope were calculated for each imputed data set, and averaged over the 10 imputed data sets.

\section{Derivation and prospective validation of the clinical risk score}

The coefficients in the model were transformed into easy-to-use risk scores by dividing all regression coefficients by the lowest coefficient value. The clinical performance of the risk scores was also evaluated. For this evaluation, we considered the test characteristics of the clinical risk score in terms of sensitivity, specificity, and positive and negative predictive values, at different categories of risk scores. The HosmerLemeshow test was used to test the goodness of fit of the risk score model.

In the prospective patient cohort, the developed clinical risk score was calculated for each patient, and sensitivity, specificity, and positive and negative predictive values were calculated at different risk score values, which were suggested as cut off values in the developmental set. Discrimination (AUC) and explained variance (Nagelkerke's $\mathrm{R}^{2}$ ) were determined in the prospective cohort.

Analysis was performed using SPSS18 (IBM, Armonk, NY, USA) and R [22]. We used versions of the MICE and RMS libraries. Requirements of the hospital research and ethical review board (Medisch Ethische Toetsingscommissie) were met, including patient informed consent.

\section{Results}

\section{Patient characteristics}

Between April 1998 and July 2010, 300 PAH and 80 LHF patients were diagnosed. Patient characteristics and haemodynamics at diagnosis in LHF and PAH patients are defined in table 1.

\section{Logistic regression, model and LHF risk score}

Univariate logistic regression was used descriptively and results are presented in table 2. Using backward regression, a medical history of left heart disease (LHD; yes=1, no=0), the sum of SV1 and RV6 on electrocardiography (ECG; in millimetres), the presence of left atrial dilation on echocardiography (LAD; yes $=1$, no $=0$ ) and the presence of left valvular heart disease that was worse than mild on echocardiography (LVD; yes=1, no=0) were identified as predictors of LHF (table 3). After correction for optimism (after boot strapping) the following model was constructed:

$$
-5.22+(2.26 \times \mathrm{LHD})+(0.10 \times \mathrm{ECG})+(2.08 \times \mathrm{LAD})+(2.28 \times \mathrm{LVD})
$$

This model had high predictive value with $\mathrm{R}^{2}=0.67$ and an AUC of 0.93 . After correction for optimism, these values were 0.65 and 0.93 , respectively (fig. 1). From the model, a LHF risk scoring system was derived (table 3). The LHF risk scores resulted in similar predictive characteristics $\left(R^{2}=0.66\right.$, AUC 0.93). Goodness of fit of the risk score model was confirmed by the Hosmer-Lemeshow test $(p=0.99)$. Table 4 shows the performance of the risk score model in terms of sensitivity, specificity, and positive and negative predictive values at different risk score categories. In figure 2, the numbers of true and false positives and true and false negatives in the same risk score categories are presented. Using a risk score cut-off value of 
$\geqslant 72$, LHF could be diagnosed noninvasively in $44 \%$ (95\% CI $33-55 \%$ ) of patients, with a PPV of $100 \%$ (95\% CI $88-100 \%)$ and $100 \%$ (95\% CI $98-100 \%)$ specificity. Alternatively, using a cut-off value of $\geqslant 64$, LHF was diagnosed noninvasively in 53\% (95\% CI $42-63 \%$ ), with a PPV of $98 \%$ (95\% CI $87-100 \%)$ and

\begin{tabular}{|c|c|c|c|}
\hline & PAH & LHF & p-value \\
\hline Patients n & 300 & 80 & \\
\hline Age years & $51.1 \pm 16.6$ & $64.2 \pm 13.8$ & $<0.001$ \\
\hline Males & $27(22-32)$ & $36(27-47)$ & 0.09 \\
\hline BMI $\mathrm{kg} \cdot \mathrm{m}^{-2}$ & $25.5 \pm 5.5$ & $28.1 \pm 5.8$ & $<0.001$ \\
\hline \multicolumn{4}{|l|}{ Medical history } \\
\hline Diabetes & $10(7-14)$ & $30(21-41)$ & $<0.001$ \\
\hline Hypertension & $19(15-24)$ & $41(31-52)$ & $<0.001$ \\
\hline Dyslipidaemia & $10(7-14)$ & $33(23-43)$ & $<0.001$ \\
\hline Smoking >1 pack-year & $47(41-53)$ & $59(48-69)$ & 0.08 \\
\hline Left valvular disease worse than mild & $2(1-4)$ & $43(32-53)$ & $<0.001$ \\
\hline Coronary artery disease & $7(4-10)$ & $31(22-42)$ & $<0.001$ \\
\hline Left heart disease & $8(6-12)$ & $58(47-68)$ & $<0.001$ \\
\hline \multicolumn{4}{|l|}{ ECG } \\
\hline Left atrial dilation & $9(7-13)$ & $5(2-13)$ & 0.27 \\
\hline $\mathrm{SV} 1+\mathrm{RV} 6 \mathrm{~mm}$ & $10.8 \pm 5.5$ & $17.3 \pm 9.0$ & $<0.001$ \\
\hline \multicolumn{4}{|l|}{ Echocardiography } \\
\hline Left atrial dilation & $24(20-29)$ & $86(77-92)$ & $<0.001$ \\
\hline Left valvular disease worse than mild & $9(7-13)$ & $65(54-75)$ & $<0.001$ \\
\hline \multicolumn{4}{|l|}{ Right heart catheterisation } \\
\hline Right atrial pressure $\mathrm{mmHg}$ & $9 \pm 9$ & $12 \pm 7$ & 0.08 \\
\hline PAP $\mathrm{mmHg}$ & $48 \pm 15$ & $42 \pm 12$ & 0.02 \\
\hline Cardiac output L.min ${ }^{-1}$ & $5.09 \pm 2.09$ & $5.37 \pm 2.18$ & 0.45 \\
\hline Heart rate beats $\cdot \min ^{-1}$ & $81 \pm 14$ & $80 \pm 17$ & 0.74 \\
\hline PVR dyn $\cdot \mathrm{s} \cdot \mathrm{cm}^{-5}$ & $792 \pm 545$ & $435 \pm 323$ & $<0.01$ \\
\hline Wedge pressure $\mathrm{mmHg}$ & $9 \pm 5$ & $21 \pm 6$ & $<0.001$ \\
\hline TPG $\mathrm{mmHg}$ & $40 \pm 16$ & $21 \pm 12$ & $<0.001$ \\
\hline Mixed venous $\mathrm{O}_{2}$ saturation $\%$ & $65 \pm 11$ & $64 \pm 10$ & 0.65 \\
\hline
\end{tabular}

Data are presented as mean \pm SD or $\%(95 \%$ CI) unless otherwise stated. BMI: body mass index; SV1: S deflection in V1; RV6: R deflection in V6; PAP: pulmonary artery pressure; PVR: pulmonary vascular resistance; TPG: transpulmonary gradient.

\begin{tabular}{|c|c|c|}
\hline & OR $(95 \% \mathrm{CI})$ & p-value \\
\hline Male sex & $1.56(0.92-2.64)$ & 0.09 \\
\hline Age per 5 years & $1.32(1.20-1.45)$ & $<0.001$ \\
\hline BMI per $5 \mathrm{~kg} \cdot \mathrm{m}^{-2}$ & $1.45(1.17-1.80)$ & $<0.01$ \\
\hline \multicolumn{3}{|l|}{ History of } \\
\hline Hypertension & $2.88(1.69-4.90)$ & $<0.001$ \\
\hline Diabetes & $3.99(2.16-7.36)$ & $<0.001$ \\
\hline Smoking & $1.57(0.94-2.63)$ & 0.08 \\
\hline Dyslipidaemia & $4.32(2.37-7.88)$ & $<0.001$ \\
\hline Coronary artery disease & $6.26(3.25-12.06)$ & $<0.001$ \\
\hline Left valve disease worse than mild & $34.55(13.76-86.72)$ & $<0.001$ \\
\hline Left heart disease & $14.88(8.14-27.21)$ & $<0.001$ \\
\hline \multicolumn{3}{|l|}{ ECG } \\
\hline Left atrial dilation & $0.55(0.18-1.63)$ & 0.28 \\
\hline $\mathrm{SV} 1+\mathrm{RV} 6$ per $5 \mathrm{~mm}$ & $1.96(1.59-2.42)$ & $<0.001$ \\
\hline \multicolumn{3}{|l|}{ Echocardiography } \\
\hline Left atrial dilation & $19.92(7.20-55.11)$ & $<0.001$ \\
\hline Left valve disease worse than mild & $18.55(7.87-43.72)$ & $<0.001$ \\
\hline
\end{tabular}


TABLE 3 Results from backward binary logistic regression, identifying independent predictors of left heart failure (LHF) and the subsequently derived LHF risk scoring system

\begin{tabular}{|c|c|c|c|}
\hline & OR $(95 \% \mathrm{CI})$ & p-value & Risk score ${ }^{\#}$ \\
\hline \multicolumn{4}{|l|}{ History of } \\
\hline Left heart disease & $10.0(4.0-25.2)$ & $<0.001$ & 22 \\
\hline \multicolumn{4}{|l|}{ ECG } \\
\hline SV1+RV6 per mm & $1.12(1.06-1.20)$ & $<0.001$ & $1 \times(S V 1+R V 6)$ \\
\hline \multicolumn{4}{|l|}{ Echocardiography } \\
\hline Left atrial dilation & $12.2(4.1-36.1)$ & $<0.001$ & 20 \\
\hline Left valve disease worse than mild & $7.5(2.5-22.2)$ & $<0.001$ & 22 \\
\hline
\end{tabular}

SV1: S deflection in V1; RV6: R deflection in V6. " : total risk score is calculated as follows. For presence of a medical history of left heart disease, the patient is attributed 22 points. If echocardiographic left atrial dilation is present, an additional 20 points are scored, and if echocardiographic left valvular disease that is worse than mild is present, an additional 22 points are scored. SV1+RV6 on ECG in millimetres is the risk score attributed for the ECG in each patient. The total score in each patient constitutes the LHF risk score for that individual.

$100 \%$ (95\% CI 98-100\%) specificity. Using the risk score with a $\geqslant 72$ cut-off, no PAH patients were falsely classified as LHF, while using a $\geqslant 64$ cut-off, one in $300(0.3 \%, 95 \%$ CI $0-2 \%)$ PAH patients were falsely classified as having LHF.

\section{Risk scores and wedge pressures}

Patients were divided into the following categories according to wedge pressure at diagnosis: $<10,10-15$, 16-20 and $>20 \mathrm{mmHg}$. Risk scores were calculated for patients in these groups. Mean \pm SD (median) risk scores were $19 \pm 15$ (12), $23 \pm 18$ (16), 54 \pm 30 (56) and $57 \pm 21$ (61), respectively (fig. 3).

\section{Prospective validation}

In the prospective validation cohort, 134 patients (79 PAH and 55 LHF) attended our clinic between August 2010 and December 2012. Mean \pm SD (median) risk scores were $23 \pm 16$ (21) and $46 \pm 24$ (40), respectively. Using the proposed risk score cut off $\geqslant 72$, PPV remained $100 \%$ (95\% CI 70-100\%), and specificity was $100 \%$ (95\% CI 94-100\%), sensitivity 20\% (95\% CI 11-33\%) and NPV 64\% (95\% CI 55$72 \%)$. At this cut-off, LHF was diagnosed noninvasively in $20 \%$, without misclassifying any PAH. Using a cut off score $\geqslant 64$, PPV was 92\% (95\% CI 65-100\%), specificity 99\% (95\% CI 93-100\%), and sensitivity and NPV 22\% (95\% CI 13-35\%) and 64\% (95\% CI 56-72\%), respectively. AUC in the prospective cohort was 0.80 (95\% CI $0.72-0.87)$ and Nagelkerke's $\mathrm{R}^{2}=0.36$.

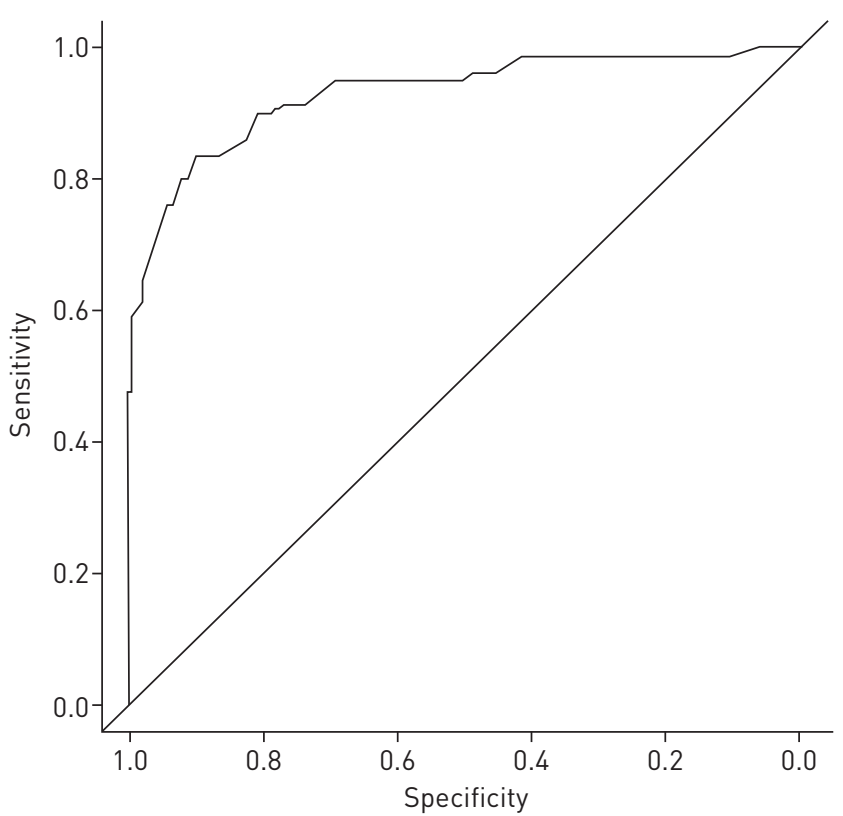

FIGURE 1 Receiver operator characteristic curve of the risk score for prediction of left heart failure in a population referred on suspicion of pulmonary arterial hypertension. 
TABLE 4 Sensitivity, specificity, positive predictive value (PPV) and negative predictive value (NPV) at various cut-off points using the risk score system, derived from the model

\begin{tabular}{lcccc} 
Risk score & Sensitivity & Specificity & PPV & NPV \\
\hline$\geqslant \mathbf{0}$ & 100 & 0 & 21 & NA \\
$\geqslant \mathbf{8}$ & 99 & 25 & 26 & 99 \\
$\geqslant \mathbf{1 6}$ & 95 & 55 & 36 & 98 \\
$\geqslant \mathbf{2 4}$ & 95 & 64 & 41 & 98 \\
$\geqslant \mathbf{3 2}$ & 91 & 77 & 51 & 97 \\
$\geqslant \mathbf{4 0}$ & 84 & 89 & 67 & 95 \\
$\geqslant \mathbf{4 8}$ & 78 & 93 & 74 & 94 \\
$\geqslant \mathbf{5 6}$ & 64 & 98 & 90 & 91 \\
$\geqslant \mathbf{6 4}$ & 51 & 100 & 98 & 89 \\
$\geqslant \mathbf{7 2}$ & 44 & 100 & 100 & 87 \\
$\geqslant \mathbf{8 0}$ & 24 & 100 & 100 & 83 \\
$\geqslant \mathbf{9 6}$ & 5 & 100 & 100 & 80 \\
& 0 & 100 & 100 & 79 \\
\hline
\end{tabular}

Data are presented as \%. NA: not applicable.

\section{Discussion}

Risk factors for heart failure are well known from the literature [9, 10]. Current LHF guidelines recommend diagnosing LHF using patient history, physical examination, laboratory, ECG and echocardiographic measures $[7,12]$. Whether these algorithms apply in the setting of $\mathrm{PH}$ is unknown. In $\mathrm{PH}$, signs of LHF might be mimicked on echocardiography due to underfilling of the left ventricle. This is especially the case for diastolic LHF [23]. Therefore, a substantial proportion of patients referred to a PAH centre has LHF as an underlying cause. Signs that can unmask the presence of an underlying LHF are left atrial enlargement and left ventricular hypertrophy (LVH) on echocardiography [6]. However, the predictive value of these parameters as a single measurement or in combination with medical history data is insufficient [24], in a setting where other signs of LHF are absent. For this reason, wedge pressure or LVEDP measurement are demanded to confirm the diagnosis of LHF as a cause of PH invasively. Any effort made to improve referral patterns to PAH centres should not lead to missed PAH diagnosis in any patient, since early detection and treatment of $\mathrm{PAH}$ improves exercise capacity and pulmonary haemodynamics [25] and delayed start of PAH-specific medications decreases survival even with a short interval [26]. Incidence rates of LHF are high, whereas PAH remains a rare disease, which further challenges a correct diagnosis by non-invasive means $[27,28]$. Our results show that in a group of patients

FIGURE 2 Pyramid graph of patients with left heart failure (LHF) and pulmonary arterial hypertension $(\mathrm{PAH})$, divided according to the total risk score outcomes of individual patients.

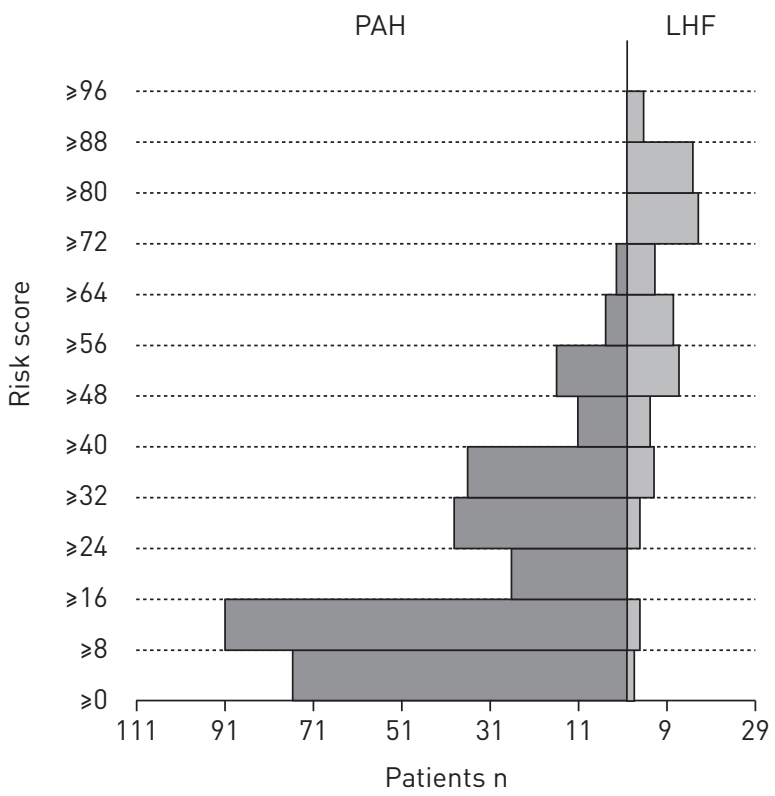




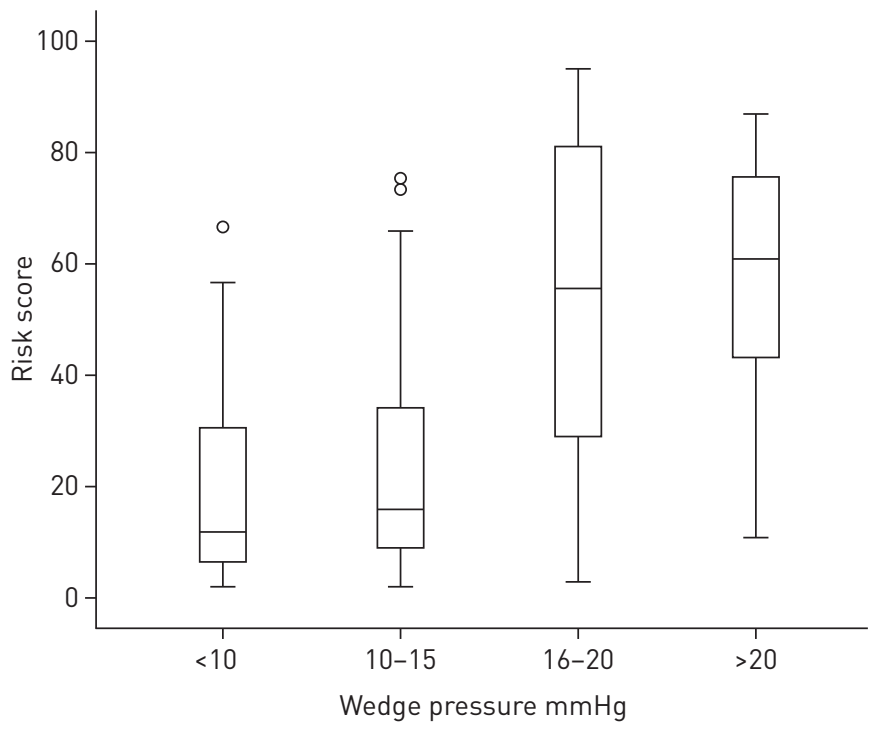

FIGURE 3 Box and whisker plots of the risk scores in patient groups with different wedge pressures at right heart catheterisation. Borders of boxes are the 25th and 75th percentile. All measurements are between the whiskers, except for outliers (circles), which are more than 1.5 times the height of the box above the 75th percentile. Median values are depicted as horizontal lines within the boxes.

referred with a diagnosis of PAH, by means of a set of easy-to-use parameters from medical history, echocardiography and ECG, LHF can be diagnosed noninvasively in a substantial number of patients.

From our data, coronary artery disease and left valvular heart disease were independent LHF predictors. This is not surprising, since both were important predictors in the Framingham and National Health and Nutrition Examination Survey I studies [9, 10]. Coronary artery disease is the most common cause of LHF [12]. Left heart disease leads to heart failure and subsequent PH only through increased left ventricular filling pressures, causing subsequent mitral insufficiency, increased left atrial filling pressures, and left atrial dilation and subsequent pulmonary venous hypertension [11]. ECG evidence of LVH had well predictive characteristics. $\mathrm{LVH}$ is a feature of left heart disease, whereas in $\mathrm{PAH}$, left ventricular atrophy can be present [29]. Left atrial dilation on MRI has high predictive value when comparing PAH and left heart failure [30]. However, echocardiography underestimates cardiovascular magnetic resonance-derived left atrial volume [31]. This explains why echocardiographic left atrial dilation, though more frequently present in pulmonary venous hypertension, has insufficient discriminatory power as a single parameter [24, 32]. ECG evidence of left atrial dilation was not significantly correlated with LHF. This may be due to suboptimal correlation with echocardiographic left atrial dilation [33, 34], further affected by nonspecific $\mathrm{P}$-wave changes due to severe right-sided atrial strain. It should be stressed that for accurate noninvasive predictions, the data from medical history, ECG and echocardiography need to be combined.

Although our study is limited by its single-centre design and our model was developed from a historical cohort, we internally validated our model by bootstrapping and performed a prospective validation study, which validated our results for external use. The echocardiography parameters were measured by a cardiologist blinded to the final diagnosis, according to current guidelines, and are widely used. Therefore, it is unlikely that these observations were operator dependent. The proportion of LHF in our study is substantial, but still relatively low compared to nontertiary secondary referral centres or primary care settings [28]. Since predictive values are strongly dependent on disease prevalence in the patient cohort studied, the higher PPV for LHF is to be expected at lower patient risk scores in settings with a smaller $\mathrm{PAH}$ prevalence. Validation in community hospitals is warranted before use of the model is recommended outside PH centres. BondERMAn et al. [35] developed a decision tree to exclude PAH, relying on the Chi-squared automatic interaction detection procedure; two out of 28 clinical, echocardiographic or ECG parameters were automatically identified (right ventricular strain on ECG, defined as ST-segment deviation and $\mathrm{T}$-wave inversion in leads $\mathrm{V} 1-\mathrm{V} 3$; and $\mathrm{N}$-terminal pro-brain natriuretic protein (NT-proBNP) with a cut off of $80 \mathrm{pg} \cdot \mathrm{mL}^{-1}$ ). BONDERMAN et al. [35] showed that in the absence of right ventricular strain and, subsequently, an NT-proBNP level $\leqslant 80 \mathrm{pg} \cdot \mathrm{mL}^{-1}$, PAH can be excluded. Our model differs from the study by BONDERMAN et al. [35], as we specifically aimed to identify LHF as alternative cause of $\mathrm{PH}$, whereas the model of BONDERMAN et al. [35] aimed to exclude PAH and included patients with normal pulmonary pressures. Considering this, and as logistic regression models have better predictive characteristics compared to regression trees, the model of BONDERMAN et al. [35] and our own model may have additional value in reducing the need for RHC [36]. The LHF patient cohort in our study displays specific characteristics, with relatively elevated PVR and pulmonary artery pressures. From the REVEAL registry, a cohort with a clinical diagnosis of PAH was described with elevated wedge pressures 
between 16 and $18 \mathrm{mmHg}$ who nonetheless, in many ways, behaved like pure group 1 patients [37]. The flip side is the recognition that some patients with heart failure with preserved ejection fraction, in particular those on diuretic medications, may have a wedge pressure of $\leqslant 15 \mathrm{mmHg}$ and an elevated PVR at the time of RHC. This phenomenon may be recognised with volume loading [15] or invasive exercise testing [38]. Even though the final diagnosis was reached after discussion by an expert panel and volume loading was routinely performed at high normal wedge pressures $(12-15 \mathrm{mmHg})$, we cannot exclude that an occasional patient was misclassified. Medicine is rarely black and white, and occasional patients present with many characteristics of group $1 \mathrm{PH}$, but may be considered a comorbidity of left heart disease (e.g. marked elevation of PVR, scleroderma, older age with hypertension and a component of heart failure with preserved ejection fraction). Such a patient may well benefit from referral to a tertiary PH centre for thoughtful discussion about management strategies. In addition, we emphasise that RHC may remain useful in selected LHF patients (e.g. in risk evaluation before valvular surgery or heart transplantation).

The advantage of the approach used in our study is that it does not require sophisticated measures. More complex echocardiographic parameters, such as the ratio of mitral velocity to early diastolic velocity of the mitral annulus, were not included not only to provide for simplicity of the model, but also because left atrial dilation measured by echocardiography had better ROCs for determining the presence or absence of diastolic dysfunction in an earlier study [39]. In addition, a notching pattern in the right ventricular outflow tract Doppler flow velocity envelope was not included as a predictor, as earlier studies showed this has insufficient predictive value in excluding PAH [40]. To conclude, our data show that LVH on ECG, left atrial dilation and left valvular heart disease on echocardiography, and medical history data of coronary artery disease and past valvular heart disease, measured in combination, can be used in a noninvasive prediction model of LHF, when echocardiography is inconclusive in excluding LHF as cause of PH. Using simple clinical parameters LHF can be diagnosed with a high level of certainty. In addition, although RHC remains necessary in case of doubt, the number of RHC can be reduced. Finally, although our data require validation in a nonreferral setting, they might be of help for the referring specialist to select patients.

\section{References}

1 Simonneau G, Galiè N, Rubin LJ, et al. Clinical classification of pulmonary hypertension. J Am Coll Cardiol 2004; 43: $1 S-12 S$.

2 D'Alonzo GE, Barst RJ, Ayres SM, et al. Survival in patients with primary pulmonary hypertension. Results from a national prospective registry. Ann Intern Med 1991; 115: 343-349.

3 Galiè N, Hoeper MM, Humbert M, et al. Guidelines for the diagnosis and treatment of pulmonary arterial hypertension: the Task Force for the Diagnosis and Treatment of Pulmonary Hypertension of the European Society of Cardiology (ESC) and the European Respiratory Society (ERS), endorsed by the International Society of Heart and Lung Transplantation (ISHLT). Eur Heart J 2009; 30: 2493-2537.

4 Badesch DB, Champion HC, Sanchez MA, et al. Diagnosis and assessment of pulmonary arterial hypertension. J Am Coll Cardiol 2009; 54: Suppl. 1, S55-S66.

5 Oudiz RJ. Pulmonary hypertension associated with left-sided heart disease. Clin Chest Med 2007; 28: 233-241.

6 Hoeper MM, Barbera JA, Channick RN, et al. Diagnosis, assessment and treatment of non-pulmonary arterial hypertension pulmonary hypertension. J Am Coll Cardiol 2009; 54: Suppl. 1, S85-S96.

7 Paulus WJ, Tschope C, Sanderson JE, et al. How to diagnose diastolic heart failure: a consensus statement on the diagnosis of heart failure with normal left ventricular ejection fraction by the Heart Failure and Echocardiography Associations of the European Society of Cardiology. Eur Heart J 2007; 28: 2539-2550.

8 Hoeper MM, Lee SH, Voswinckel R, et al. Complications of right heart catheterisation procedures in patients with pulmonary hypertension in experienced centers. J Am Coll Cardiol 2006; 48: 2546-2552.

9 Kannel WB, D’Agustino RB, Silbershatz H, et al. Profile for estimating risk of heart failure. Arch Intern Med 1999; 159: 1197-1204.

10 He J, Ogden LG, Bazzamo LA, et al. Risk factors for congestive heart failure in US men and women. NHANES I epidemiologic follow-up study. Arch Intern Med 2001; 161: 996-1002.

11 Leung DY, Boyd A, Ng AA, et al. Echocardiographic evaluation of left atrial size and function: current understanding, pathophysiologic correlates, and prognostic implications. Am Heart J 2008; 156: 1056-1064.

12 Dickstein K, Cohen-Solal A, Filippatos G, et al. ESC guidelines for the diagnosis and treatment of acute and chronic heart failure 2008. The Task Force for the Diagnosis and Treatment of the European Society of Cardiology. Developed in collaboration with the Heart Failure Association of the ESC (HFA) and endorsed by the European Society of Intensive Care Medicine (ESICM). Eur Heart J 2008; 29: 2388-2442.

13 Fox KF, Cowie MR, Wood DA, et al. Coronary artery disease as the cause of incident heart failure in the population. Eur Heart J 2001; 22: 228-236.

14 McLaughlin VV, Badesch DB, Barst RJ, et al. ACCF/AHA 2009 expert consensus document on pulmonary hypertension a report of the American College of Cardiology Foundation Task Force on Expert Consensus Documents and the American Heart Association developed in collaboration with the American College of Chest Physicians; American Thoracic Society, Inc.; and the Pulmonary Hypertension Association. J Am Coll Cardiol 2009; 53: 1573-1619.

15 Robbins IM, Hemnes AR, Pugh ME, et al. High prevalence of occult pulmonary venous hypertension revealed by fluid challenge in pulmonary hypertension. Circ Heart Fail 2014; 7: 116-122.

16 Lang RM, Bierig M, Devereux RB, et al. Recommendations for chamber quantification. Eur J Echocardiogr 2006 7: 79-108.

17 Lancellotti P, Moura L, Pierard LA, et al. European association of echocardiography recommendations for the assessment of valvular regurgitation. Part 2: mitral and tricuspid regurgitation (native valve disease). Eur J Echocardiogr 2010; 11: 307-332. 

recommendations for clinical practice. Eur J Echocardiogr 2009; 10: 1-25.

19 Lancellotti P, Tribouilloy C, Hagendorff A, et al. European association of echocardiography recommendations for the assessment of valvular regurgitation. Part 1: aortic and pulmonary regurgitation (native valve disease). Eur J Echocardiogr 2010; 11: 223-244.

20 Wood AM, White IR, Royston P. How should variable selection be performed with multiply imputed data? Stat Med 2008; 27: 3227-3246.

21 Harrel FE. Regression Modeling Strategies. Berlin, Springer, 2001.

22 R Development Core Team 2010. R: a Language and Environment for Statistical Computing. Vienna, R Foundation for Statistical Computing, 2010.

23 Shapiro BP, Nishimura RA, McGoon MD, et al. Diagnostic dilemmas: diastolic heart failure causing pulmonary hypertension and pulmonary hypertension causing diastolic dysfunction. Adv Pulm Hypertens 2006; 5: 13-20.

24 Thenappan T, Shah S, Gomberg-Maitland M, et al. Clinical characteristic of pulmonary hypertension in patients with heart failure and preserved ejection fraction. Circ Heart Fail 2011; 4: 257-265.

25 Galiè N, Rubin LJ, Hoeper MM, et al. Treatment of patients with mildly progressive pulmonary arterial hypertension with bosentan (EARLY study): a double-blind, randomised controlled trial. Lancet 2008; 371: 2093-2100.

26 Barst RJ, Rubin LJ, Long WA, et al. A comparison of continuous intravenous epoprostenol (prostacyclin) with conventional therapy for primary pulmonary hypertension. N Engl J Med 1996; 334: 296-302.

27 Bleumink GS, Knetsch AM, Sturkenboom MC, et al. Quantifying the heart failure epidemic: prevalence, incidence rate, lifetime risk and prognosis of heart failure. The Rotterdam study. Eur Heart J 2004; 25: 1614-1619.

28 Gabbay E, Yeow W, Playford D, et al. Pulmonary arterial hypertension (PAH) is an uncommon cause of pulmonary hypertension $(\mathrm{PH})$ in an unselected population: the Armadale echocardiography study. Am J Respir Crit Care Med 2007; 175: A713.

29 Hardziyenka M, Campian ME, Reesink HJ, et al. Right ventricular failure following chronic pressure overload is associated with reduction in left ventricular mass: evidence for atrophic remodeling. J Am Coll Cardiol 2011; 57: 921-928.

30 Crawley SF, Johnson MK, Dargie HJ, et al. LA volume by CMR distinguishes idiopathic from pulmonary hypertension due to HFpEF. JACC Cardiovasc Imaging 2013; 6: 1120-1121.

31 Whitlock M, Garg A, Gelow J, et al. Comparison of left and right atrial volume by echocardiography versus cardiac magnetic resonance imaging using the area-length method. Am J Cardiol 2010; 106: 1345-1350.

32 Brittain EL, Pugh ME, Wang L, et al. Predictors of diastolic-to-wedge gradient in patients evaluated for pulmonary hypertension. PLoS One 2013; 8: e76461.

33 Waggoner AD, Adyanthaya AV, Quinones MA, et al. Left atrial enlargement. Echocardiographic assessment of electrocardiographic criteria. Circulation 1976; 54: 553-557.

34 Lee KS, Appleton CP, Lester SJ, et al. Relation of electrocardiographic criteria for left atrial enlargement to two-dimensional echocardiographic left atrial volume measurements. Am J Cardiol 2007; 99: 113-118.

35 Bonderman D, Wexberg P, Martischnig AM, et al. A noninvasive algorithm to exclude pre-capillary pulmonary hypertension. Eur Respir J 2011; 37: 1096-1103.

36 Austin PC. A comparison of regression trees, logistic regression, generalized additive models, amd multivariate adaptive regression splines for predicting AMI mortality. Stat Med 2007; 26: 2937-2957.

37 Frost AE, Farber HW, Barst RJ, et al. Demographics and outcomes of patients diagnosed with pulmonary hypertension with pulmonary capillary wedge pressures 16 to $18 \mathrm{~mm} \mathrm{Hg}$ : insights from the REVEAL Registry. Chest 2013; 143: 185-195.

38 Borlaug BA, Nishimura RA, Sorajja P, et al. Exercise hemodynamics enhance diagnosis of early heart failure with preserved ejection fraction. Circ Heart Fail 2010; 3: 588-595.

39 Tsang TS, Barnes ME, Gersh BJ, et al. Left atrial volume as a morphophysiologic expression of left ventricular diastolic dysfunction and relation to cardiovascular risk burden. Am J Cardiol 2002; 90: 1284-1289.

40 Arkles JS, Opotowsky AR, Ojeda J, et al. Shape of the right ventricular Doppler envelope predicts hemodynamics and right heart function in pulmonary hypertension. Am J Respir Crit Care Med 2011; 183: 268-276. 